\title{
Pinning Cluster Synchronization in Linear Hybrid Coupled Delayed Dynamical Networks
}

\author{
Anping Bao, ${ }^{1}$ Ting Wang, ${ }^{2}$ Shumin Fei, ${ }^{1}$ and Xiaomin Tian' \\ ${ }^{1}$ Key Laboratory of Measurement and Control of CSE (School of Automation, Southeast University), Ministry of Education, \\ Nanjing 210096, China \\ ${ }^{2}$ School of Information Science and Technology, Nanjing Forestry University, Nanjing 210037, China
}

Correspondence should be addressed to Anping Bao; baoanpingseu@163.com

Received 30 December 2015; Accepted 17 April 2016

Academic Editor: Xiangyu Meng

Copyright (c) 2016 Anping Bao et al. This is an open access article distributed under the Creative Commons Attribution License, which permits unrestricted use, distribution, and reproduction in any medium, provided the original work is properly cited.

\begin{abstract}
The problem on cluster synchronization will be investigated for a class of delayed dynamical networks based on pinning control strategy. Through utilizing the combined convex technique and Kronecker product, two sufficient conditions can be derived to ensure the desired synchronization when the designed feedback controller is employed to each cluster. Moreover, the inner coupling matrices are unnecessarily restricted to be diagonal and the controller design can be converted into solving a series of linear matrix inequalities (LMIs), which greatly improve the present methods. Finally, two numerical examples are provided to demonstrate the effectiveness and reduced conservatism.
\end{abstract}

\section{Introduction}

In past decade, the synchronization of various chaotic systems has received considerable attention since the pioneering works have appeared $[1,2]$. Presently, it is widely known that many benefits of having synchronization can be existent. In particular, the synchronization in language emergence and development results can come up with the common vocabulary and agents' synchronization in organization management can improve their work efficiency. Thus recently, the synchronization has been widely studied owing to its great potential applications. Furthermore, since chaos synchronization in arrays of coupled dynamical networks was initially studied [3], various coupled networks have received the attention because they can exhibit some interesting phenomena $[4,5]$, and many elegant results have been reported [6-32]. In particular, in $[6,7,33]$, time-delay is unavoidable and delayed neural networks (DNNs) are verified to exhibit some complex and unpredictable behaviors, such as periodic oscillations, bifurcation, and chaotic attractors; then, the impulsive and adaptive synchronization has also been studied [8-11], and some uneasy-to-test results have been presented. Most recently, through using Kronecker product, the global synchronization has been studied and elegant criteria have been obtained in terms of LMIs [12-20, 23]. Yet it is worth noting that, in the above works, some most developed techniques were not utilized and the addressed networks seemed to be of simple forms. Thus, researchers have used some effective tools to give less conservative results ensuring the synchronization for more general coupled DNNs [23].

In 1992, as the truth that the effective coupling among neurons varies temporally in a rather short time scale has been found [34], some researchers have mentioned that the degree of synchronization among pairs of neurons changed both temporally and by the choice of pairs. Therefore, the cluster synchronization has been imposed to various dynamical networks [21, 22, 24-31, 35]. However, due to the existence of embedding of invariant synchronization manifolds, it may occur that the system can reach different clustering patterns from the different initial conditions [24-29]. Thus, together with pinning control, some suitable methods have appeared but have been independent of initial states [30]. In [30], the pinning control strategy has been used to realize the cluster synchronization for stochastic coupled DNNs, in which the upper bound of delay variation was less than 1. Later, some effective techniques were used to overcome 
the shortcoming during tackling the delayed dynamical networks $[31,35]$. Yet though these results above were elegant, there still exist some points waiting for the improvements. Firstly, most works above have not contained lower bound of delay variation and, in fact, its information can play an important role in reducing the conservatism. Secondly, in $[30,31]$, the inner coupling matrices had to be diagonal, which unavoidably limits the application areas. Thirdly, as for delay $\tau(t) \in\left[\tau_{0}, \tau_{m}\right]$, since the triple integral LKF terms such as $\left(\left(\tau_{m}^{2}-\tau_{0}^{2}\right) / 2\right) \int_{-\tau_{m}}^{-\tau_{0}} \int_{\varrho}^{0} \int_{t+\theta}^{t} \dot{x}^{T}(s) Q \dot{x}(s) d s d \theta d \varrho$ were firstly put forward [35], it has been used and improved owing to the fact that it could help reduce the conservatism greatly [36]. Yet the authors noticed that some important terms have been ignored when estimating its derivative $[35,36]$, which also induces the conservatism. Therefore, the tighter estimation should be given. Overall, as for the pinning cluster synchronization of coupled networks, the mentioned points above have not been considered, which remains important and motivates this work.

Inspired by the above discussions, this paper aims to study the problem on cluster synchronization for a class of coupled time-delay networks with linear hybrid coupling by means of pinning control. Through choosing two augmented Lyapunov-Krasovskii functionals (LKFs) and using the combined convex technique, some novel sufficient conditions are presented via Kronecker product and LMIs, whose feasibility can be easily checked by resorting to Matlab LMI Toolbox. In particular, we will give the tighter upper bounds on time derivative of LKF terms. The efficiency and less conservatism can be verified on the basis of two numerical examples.

Notations. $\mathbf{R}^{n \times m}$ is the set of all $n \times m$ real matrices; $I_{m}$ represents the $m \times m$ identity matrix and $0_{m \cdot n}$ denotes the $m \times n$ zero matrix; $A \otimes B$ represents Kronecker product of matrices $A$ and $B$.

\section{Problem Formulations and Preliminaries}

Firstly, suppose the nodes are coupled with states $x_{i}(t)$, $i \in\{1, \ldots, N\}$; we consider the dynamical networks with each node being an $n$-dimensional DNN with linear hybrid coupling as

$$
\begin{aligned}
\dot{x}_{i}(t)= & -C x_{i}(t)+A \bar{f}\left(x_{i}(t)\right)+B \bar{f}\left(x_{i}(t-\tau(t))\right) \\
& +I(t)+u_{i}(t)+\sum_{j=1}^{N} l_{i j}^{1} G x_{j}(t) \\
& +\sum_{j=1}^{N} l_{i j}^{2} H x_{j}(t-\tau(t)) \\
& +\sum_{j=1}^{N} l_{i j}^{3} K\left(\int_{t-\tau(t)}^{t} x_{j}(s) d s\right),
\end{aligned}
$$

where $x_{i}(t)=\left[x_{i 1}(t), x_{i 2}(t), \ldots, x_{i n}(t)\right]^{T}$ are the state vectors; here $C=\operatorname{diag}\left(c_{1}, c_{2}, \ldots, c_{n}\right)>0, A=\left[a_{i j}\right]_{n \times n}, B=\left[b_{i j}\right]_{n \times n}$, and $\bar{f}\left(x_{i}(\cdot)\right)=\left[\bar{f}_{1}\left(x_{i 1}(\cdot)\right), \ldots, \bar{f}_{n}\left(x_{i n}(\cdot)\right)\right]^{T}$ are the activation functions; also here we assume $G=\left[g_{i j}\right]_{n \times n}, H=\left[h_{i j}\right]_{n \times n}$,
$K=\left[k_{i j}\right]_{n \times n}$ denote the inner coupling matrices, $u_{i}(t)$ is the control input, and $I(t) \in \mathbf{R}^{n}$ is the input vector.

Remark 1. In system (1), the hybrid coupling is utilized in model (1) and it should be emphasized that the inner coupling matrices $G, H$, and $K$ are not necessarily restricted to be of diagonal form, which can represent more general cases than the ones in $[30,31]$.

Suppose that networks (1) will be controlled onto some desired inhomogeneous state as $\left\{x_{1}(t), \ldots, x_{m_{1}}(t)\right\} \rightarrow$ $s_{1}(t),\left\{x_{m_{1}+1}(t), \ldots, x_{m_{2}}(t)\right\} \rightarrow s_{2}(t), \ldots,\left\{x_{m_{k-1}+1}(t), \ldots\right.$, $\left.x_{m_{k}}(t)\right\} \rightarrow s_{k}(t)$; that is, $\mathscr{M}=\left\{\left\{s_{1}(t), \ldots, s_{1}(t)\right\},\left\{s_{2}(t), \ldots\right.\right.$, $\left.\left.s_{2}(t)\right\}, \ldots,\left\{s_{k}(t), \ldots, s_{k}(t)\right\}\right\} \in \mathbf{R}^{n \times N}$ is the desired cluster synchronization pattern under the pinning control, where $x_{i}(t) \rightarrow s_{l}(t)$ means that $\lim _{t \rightarrow+\infty}\left\|x_{i}(t)-s_{l}(t)\right\|=0$ for $i \in\left\{m_{l-1}, \ldots, m_{l}\right\}$ with $m_{0}=0$ and $l \in\{1, \ldots, k\}$. The function $s_{l}(t)$ is defined as

$$
\begin{aligned}
\dot{s}_{l}(t)= & -C s_{l}(t)+A \bar{f}\left(s_{l}(t)\right)+B \bar{f}\left(s_{l}(t-\tau(t))\right) \\
& +I(t), \quad l=1, \ldots, k .
\end{aligned}
$$

For the dynamical networks described by (1), the following assumptions are utilized.

(A1) Here $\tau(t)$ denotes the interval time-varying delay satisfying

$$
\begin{gathered}
0 \leq \tau_{0} \leq \tau(t) \leq \tau_{m}, \\
\mu_{0} \leq \dot{\tau}(t) \leq \mu_{m}<+\infty
\end{gathered}
$$

Moreover, we give the denotations as $\bar{\tau}_{m}=\tau_{m}-\tau_{0}, \bar{\mu}_{m}=$ $\mu_{m}-\mu_{0}$, and $\delta_{m}=\tau_{m}^{2}-\tau_{0}^{2}$.

(A2) For $v=1,2,3$ and the configuration matrices

$$
L^{\nu}=\left[\begin{array}{cccc}
L_{11}^{v} & L_{12}^{v} & \cdots & L_{1 k}^{v} \\
L_{21}^{v} & L_{22}^{v} & \cdots & L_{2 k}^{v} \\
\vdots & \vdots & \ddots & \vdots \\
L_{k 1}^{v} & L_{k 2}^{v} & \cdots & L_{k k}^{v}
\end{array}\right]
$$

with $L_{i i}^{\nu} \in R^{\left(m_{i}-m_{i-1}\right) \times\left(m_{i}-m_{i-1}\right)}$ and $L_{i j}^{\nu} \in R^{\left(m_{i}-m_{i-1}\right) \times\left(m_{j}-m_{j-1}\right)}$, $i, j \in\{1, \ldots, k\}$, assume that every matrix $L_{i i}^{v}=\left[l_{i i, g h}^{v}\right]$ for $i \in\{1, \ldots, k\}$ satisfies $l_{i i, g g}^{v} \geq 0, l_{i i, g g}^{v}=-\sum_{h=1, h \neq g}^{m_{i}-m_{i-1}} l_{i i, g h}^{v}$, and the sums of all rows in every $L_{i j}^{\nu}(i \neq j)$ are zeros.

(A3) There exist constants $\sigma_{i}^{-}, \sigma_{i}^{+} \in \mathbf{R}$ such that the bounded functions $\bar{f}_{i}(\cdot)$ satisfy

$$
\sigma_{i}^{-} \leq \frac{\bar{f}_{i}(\alpha)-\bar{f}_{i}(\beta)}{\alpha-\beta} \leq \sigma_{i}^{+}, \quad \forall \alpha, \beta \in \mathbf{R}, i=1, \ldots, n
$$

Here we set $\Sigma^{+}=\operatorname{diag}\left(\sigma_{1}^{+}, \ldots, \sigma_{n}^{+}\right), \Sigma^{-}=\operatorname{diag}\left(\sigma_{1}^{-}, \ldots, \sigma_{n}^{-}\right)$, and

$$
\begin{aligned}
& \Sigma_{1}=\operatorname{diag}\left(\sigma_{1}^{+} \sigma_{1}^{-}, \ldots, \sigma_{n}^{+} \sigma_{n}^{-}\right), \\
& \Sigma_{2}=\operatorname{diag}\left(\frac{\sigma_{1}^{+}+\sigma_{1}^{-}}{2}, \ldots, \frac{\sigma_{n}^{+}+\sigma_{n}^{-}}{2}\right) .
\end{aligned}
$$


In this paper, we consider one special case that the accurate information on time-delay is available. Without loss of generality, to achieve the goal of cluster synchronization in this work, we will apply the pinning control strategy on the nodes set $J=\left\{m_{1}, m_{2}, \ldots, m_{k}\right\}$ and adopt the following pinning controller as

$$
u_{i}(t)= \begin{cases}-l_{i} G\left[x_{i}(t)-s_{l}(t)\right]-r_{i} H\left[x_{i}(t-\tau(t))-s_{l}(t-\tau(t))\right], & i=m_{l}, l=1, \ldots, k, \\ 0, & i \neq m_{l} .\end{cases}
$$

Let $e_{i}(t)=x_{i}(t)-s_{l}(t)$; one can check that $\sum_{j=1}^{N} l_{i j}^{1} G x_{j}(\cdot)=\sum_{j=1}^{N} l_{i j}^{1} G e_{j}(\cdot), \sum_{j=1}^{N} l_{i j}^{2} H x_{j}(\cdot)=\sum_{j=1}^{N} l_{i j}^{2} H e_{j}(\cdot)$, and $\sum_{j=1}^{N} l_{i j}^{3} K x_{j}(\cdot)=\sum_{j=1}^{N} l_{i j}^{3} K e_{j}(\cdot)$. Then, combining (1) and (2) with (7) yields

$$
\begin{aligned}
\dot{e}_{i}(t)= & -\left(C+\bar{l}_{i} G\right) e_{i}(t)+A f\left(e_{i}(t)\right) \\
& +B f\left(e_{i}(t-\tau(t))\right)-\bar{r}_{i} H e_{i}(t-\tau(t)) \\
& +\sum_{j=1}^{N} l_{i j}^{1} G e_{j}(t)+\sum_{j=1}^{N} l_{i j}^{2} H e_{j}(t-\tau(t)) \\
& +\sum_{j=1}^{N} l_{i j}^{3} K\left(\int_{t-\tau(t)}^{t} e_{j}(s) d s\right),
\end{aligned}
$$

Then, we can easily check that the functions $f(\cdot)$ satisfy assumption (A3) and we set

$$
\begin{aligned}
& \Xi=\operatorname{diag}\left(\underline{0, \ldots, 0, l_{m_{1}}}, \ldots, \underline{0, \ldots, 0, l_{m_{k}}}\right), \\
& \Theta=\operatorname{diag}\left(\underline{\left.0, \ldots, 0, r_{m_{1}}, \ldots, 0, \ldots, 0, r_{m_{k}}\right) .}\right.
\end{aligned}
$$

In what follows, some useful basic definition and denotations will be introduced.

Definition 2 (see [30]). The dynamical network (1) with $N$ nodes is said to achieve the cluster synchronization, if the $N$ nodes are split into $k$ clusters $G_{1}, G_{2}, \ldots, G_{k}$ as $\left\{G_{1}=\right.$ $\left(1, \ldots, m_{1}\right), G_{2}=\left(m_{1}+1, \ldots, m_{2}\right), \ldots, G_{k}=\left(m_{k-1}+\right.$ $\left.\left.1, \ldots, m_{k}\right)\right\}$ such that the nodes synchronize with each other in the same cluster; namely, for the states $x_{i}(t)$ and $x_{j}(t)$ of the arbitrary nodes $i$ and $j$ in the same cluster $G_{l}(l=1, \ldots, k)$, $\lim _{t \rightarrow+\infty}\left\|x_{i}(t)-x_{j}(t)\right\|=0$ holds, in which $\|\cdot\|$ stands for the Euclidean norm.

Denotation 1. Denote the $3 N n \times 3 N n$ constant matrix $\mathbf{E}$ as

E

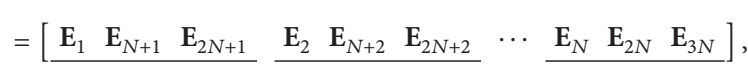

in which the $3 N n \times n$ matrix $\mathbf{E}_{i}(i=1, \ldots, 3 N)$ can be expressed as follows:

$$
\mathbf{E}_{i}^{T}=\left[\begin{array}{lllllll}
0_{n} & 0_{n} & \cdots & I_{n} & \cdots & 0_{n} & 0_{n}
\end{array}\right]
$$

with the identity matrix $I_{n}$ denoting the $i$ th one in the matrix vector $\mathbf{E}_{i}$.

Denotation 2. Denote

$$
\begin{aligned}
e^{T}(\cdot) & =\left[\begin{array}{llll}
e_{1}^{T}(\cdot) & e_{2}^{T}(\cdot) & \cdots & e_{N}^{T}(\cdot)
\end{array}\right], \\
\dot{e}^{T}(t) & =\left[\begin{array}{llll}
\dot{e}_{1}^{T}(t) & \dot{e}_{2}^{T}(t) & \cdots & \dot{e}_{N}^{T}(t)
\end{array}\right] .
\end{aligned}
$$

\section{Pinning Cluster Synchronization}

Prior to addressing the main results, the following lemmas will be useful in the proof.

Lemma 3 (see [35]). For any constant matrix $X \in \mathbf{R}^{n \times n}, X=$ $X^{T} \geq 0$, two scalars $h_{2} \geq h_{1} \geq 0$, such that the following integrations are well defined; then

$$
\begin{aligned}
& -\left(h_{2}-h_{1}\right) \int_{t-h_{2}}^{t-h_{1}} x^{T}(s) X x(s) d s \\
& \leq-\left(\int_{t-h_{2}}^{t-h_{1}} x(s) d s\right)^{T} X\left(\int_{-h_{2}}^{-h_{1}} x(s) d s\right) ; \\
& -\frac{h_{2}^{2}-h_{1}^{2}}{2} \int_{-h_{2}}^{-h_{1}} \int_{t+\theta}^{t} x^{T}(s) X x(s) d s d \theta \\
& \leq-\left(\int_{-h_{2}}^{-h_{1}} \int_{t+\theta}^{t} x(s) d s d \theta\right)^{T} \\
& \cdot X\left(\int_{-h_{2}}^{-h_{1}} \int_{t+\theta}^{t} x(s) d s d \theta\right) .
\end{aligned}
$$

Lemma 4 (see [37]). For any vectors $\zeta_{1}$ and $\zeta_{2}$, constant matrices $R$ and $S$, and real scalars $\alpha \geq 0$ and $\beta \geq 0$ satisfying that $\left[\begin{array}{ll}R & S \\ * & R\end{array}\right] \geq 0$ and $\alpha+\beta=1$, the inequality holds:

$$
-\frac{1}{\alpha} \zeta_{1}^{T} R \zeta_{1}-\frac{1}{\beta} \zeta_{2}^{T} R \zeta_{2} \leq-\left[\begin{array}{l}
\zeta_{1} \\
\zeta_{2}
\end{array}\right]^{T}\left[\begin{array}{ll}
R & S \\
* & R
\end{array}\right]\left[\begin{array}{l}
\zeta_{1} \\
\zeta_{2}
\end{array}\right] .
$$

Lemma 5 (see [23]). Suppose that $\Omega, \Xi_{1}$, and $\Xi_{2}$ are the constant matrices of appropriate dimensions, $\alpha \in[0,1]$; then $\Omega+\alpha \Xi_{1}+(1-\alpha) \Xi_{2}<0$ holds, if and only if $\Omega+\Xi_{1}<0$, $\Omega+\Xi_{2}<0$. 
Lemma 6 (see [37]). For the symmetric appropriately dimensional matrices $R>0, \Xi$, and matrix $\Gamma$, the two following statements are equivalent: (i) $\Xi-\Gamma^{T} R \Gamma<0$; (ii) there exists a matrix of appropriate dimension $\Pi$ such that

$$
\begin{gathered}
{\left[\begin{array}{cc}
\Xi+\Gamma^{T} \Pi^{T}+\Pi \Gamma & \Pi R^{-1} \\
* & -R^{-1}
\end{array}\right]<0} \\
\text { or }\left[\begin{array}{cc}
\Xi+\Gamma^{T} \Pi^{T}+\Pi \Gamma & \Pi \\
* & -R
\end{array}\right]<0 .
\end{gathered}
$$

Now, together with the pinning control strategy, two less conservative criteria will be presented for the cluster synchronization based on Kronecker product and LMI approach.

Theorem 7. Suppose that assumptions (A1)-(A3) are true; then, the controlled dynamical networks (1) can achieve the desired cluster synchronization, if there exist two $6 \mathrm{Nn} \times \mathrm{Nn}$ matrices $\Pi_{i}(i=1,2)$ making $\Pi=\left[\begin{array}{ll}\Pi_{1} & \Pi_{2}\end{array}\right], n \times n$ constant matrices $P>0, P_{i}>0(i=1,2,3,4), Q_{j}>0, R_{j}>0$, $S_{j}(j=1,2,3)$, and $M_{l}, N_{l}>0(l=1,2)$ guaranteeing $\left[\begin{array}{cc}R_{j} & S_{j} \\ * & R_{j}\end{array}\right] \geq 0$ and $n \times n$ diagonal matrices $E>0, F>0, U>0$, and $V>0$ such that the LMIs in (17)-(18) hold:

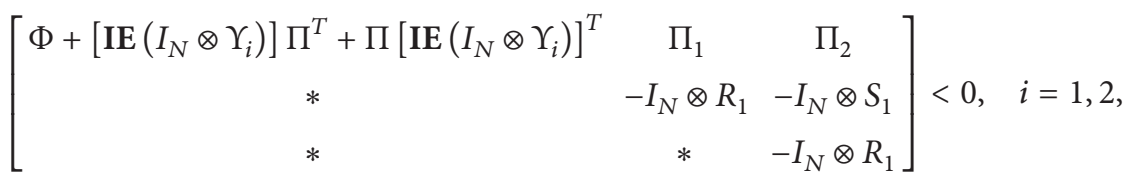

$$
\begin{aligned}
& \Omega+\bar{\mu}_{m} \Gamma_{1}\left[\begin{array}{cc}
P_{1} & 0 \\
0 & P_{3}
\end{array}\right] \Gamma_{1}^{T}<0, \\
& \Omega+\bar{\mu}_{m} \Gamma_{2} P_{4} \Gamma_{2}^{T}<0,
\end{aligned}
$$

where $\mathbf{E}$ is expressed in Denotation 1 and

$$
\begin{aligned}
\Phi & =\left[\begin{array}{cccccc}
\Phi_{11} & \Phi_{12} & \Phi_{13} & \Phi_{14} & 0 & \Phi_{16} \\
* & \Phi_{22} & 0 & 0 & 0 & \Phi_{26} \\
* & * & \Phi_{33} & 0 & 0 & \Phi_{36} \\
* & * & * & \Phi_{44} & \Phi_{45} & \Phi_{46} \\
* & * & * & * & \Phi_{55} & 0 \\
* & * & * & * & * & \Phi_{66}
\end{array}\right], \\
\Omega & =\left[\begin{array}{ccccccc}
\Omega_{11} & Q_{3} & M_{1} B & 0 & \Omega_{15} & M_{1} B & \Omega_{17} \\
* & \Omega_{22} & \Omega_{23} & S_{3} & 0 & 0 & 0 \\
* & * & \Omega_{33} & \Omega_{34} & 0 & V \Sigma_{2} & 0 \\
* & * & * & \Omega_{44} & 0 & 0 & 0 \\
* & * & * & * & \Omega_{55} & 0 & \Omega_{57} \\
* & * & * & * & * & \Omega_{66} & B^{T} M_{2}^{T} \\
* & * & * & * & * & * & \Omega_{77}
\end{array}\right],
\end{aligned}
$$$$
\mathbf{I}^{T}=\left[\begin{array}{ccc}
I_{N n} & 0_{N n \cdot 3 N n} & 0_{N n \cdot 2 N n} \\
0_{N n \cdot 3 N n} & I_{N n} & 0_{N n \cdot 2 N n} \\
0_{N n \cdot 4 N n} & I_{N n} & 0_{N n \cdot N n}
\end{array}\right],
$$$$
\Upsilon_{1}=\left[\begin{array}{cc}
\bar{\tau}_{m} I_{n} & 0_{n} \\
-I_{n} & 0_{n} \\
0_{n} & -I_{n}
\end{array}\right]
$$

$$
\begin{gathered}
\Upsilon_{2}=\left[\begin{array}{cc}
0_{n} & \bar{\tau}_{m} I_{n} \\
-I_{n} & 0_{n} \\
0_{n} & -I_{n}
\end{array}\right], \\
\Gamma_{1}=\left[\begin{array}{cc}
0_{5 n \cdot n} & 0_{3 n \cdot n} \\
I_{n} & I_{n} \\
0_{n} & 0_{3 n \cdot n}
\end{array}\right],
\end{gathered}
$$

$$
\text { and } \begin{aligned}
\Gamma_{2}= & {\left[\begin{array}{lll}
l_{n \cdot 3 n} & I_{n} & 0_{n \cdot 3 n}
\end{array}\right]^{T} \text { with } } \\
\Phi_{11}= & L^{1} \otimes\left(M_{2} G\right)+\left(L^{1} \otimes M_{2} G\right)^{T}-2 \Xi \otimes\left(M_{1} G\right) \\
& -I_{N} \otimes\left(\tau_{0}^{2} Q_{1}\right), \\
\Phi_{12}= & {\left[\left(L^{2}\right)^{T}-\Theta\right] \otimes\left(M_{1} H\right), } \\
\Phi_{13}= & I_{N} \otimes\left(\tau_{0} Q_{1}\right)+L^{3} \otimes\left(M_{1} K\right), \\
\Phi_{14}= & L^{3} \otimes\left(M_{1} K\right), \\
\Phi_{16}= & {\left[\left(L^{1}\right)^{T}-\Xi\right] \otimes\left(M_{2} G\right)^{T}, } \\
\Phi_{22}= & -I_{N} \otimes N_{2}, \\
\Phi_{26}= & {\left[\left(L^{2}\right)^{T}-\Theta\right] \otimes\left(M_{2} H\right)^{T}, } \\
\Phi_{33}= & -I_{N} \otimes\left(Q_{1}+Q_{2}\right), \\
\Phi_{36}= & \left(L^{3} \otimes M_{2} K\right)^{T},
\end{aligned}
$$




$$
\begin{aligned}
& \Phi_{44}=-I_{N} \otimes R_{2}, \\
& \Phi_{45}=-I_{N} \otimes S_{2}, \\
& \Phi_{46}=\left(L^{3} \otimes M_{2} K\right)^{T}, \\
& \Phi_{55}=-I_{N} \otimes R_{2}, \\
& \Phi_{66}=-I_{N} \otimes N_{1} \text {, } \\
& \Omega_{11}=P_{2}+\tau_{0}^{2} Q_{2}+\bar{\tau}_{m}^{2} R_{2}-Q_{3}-U \Sigma_{1}-M_{1} C \\
& -C M_{1}^{T} \text {, } \\
& \Omega_{15}=M_{1} A+U \Sigma_{2} \text {, } \\
& \Omega_{17}=P-C M_{2}^{T}-M_{1}+F \Sigma^{+}-E \Sigma^{-}, \\
& \Omega_{22}=-P_{2}-Q_{3}-R_{3}+P_{3} \text {, } \\
& \Omega_{23}=R_{3}-S_{3} \text {, } \\
& \Omega_{33}=\left(\mu_{0}-1\right) P_{3}+\left(1-\mu_{m}\right) P_{4}-2 R_{3}+S_{3}+S_{3}^{T} \\
& -V \Sigma_{1}+N_{2} \text {, } \\
& \Omega_{34}=R_{3}-S_{3} \text {, } \\
& \Omega_{44}=-P_{4}-R_{3} \text {, } \\
& \Omega_{55}=-U+P_{1} \text {, } \\
& \Omega_{57}=E^{T}-F^{T}+A^{T} M_{2}^{T}, \\
& \Omega_{66}=-V+\left(\mu_{0}-1\right) P_{1},
\end{aligned}
$$

$$
\begin{aligned}
\Omega_{77}= & -M_{2}-M_{2}^{T}+N_{1}+\tau_{0}^{2} Q_{3}+\bar{\tau}_{m}^{2} R_{3}+\frac{\tau_{0}^{4}}{4} Q_{1} \\
& +\frac{\delta_{m}^{2}}{4} R_{1} .
\end{aligned}
$$

In what follows, based on Theorem 7, we can consider the pinning cluster synchronization for the dynamical networks composed of $N$ time-delay Lur'e systems [32]:

$$
\begin{aligned}
\dot{x}_{i}(t)= & -C x_{i}(t)+A \bar{f}\left(W^{T} x_{i}(t)\right)+\sum_{j=1}^{N} l_{i j}^{1} G x_{j}(t) \\
& +\sum_{j=1}^{N} l_{i j}^{2} H x_{j}(t-\tau(t))+u_{i}(t),
\end{aligned}
$$

where $C=\left[a_{i j}\right]_{n \times n}, A=\left[c_{i j}\right]_{n \times n_{1}}$, and $W=\left[w_{i j}\right]_{n \times n_{1}}=$ $\left[W_{1}, W_{2}, \ldots, W_{n_{1}}\right]$ are constant matrices; here $\bar{f}\left(W^{T} x(t)\right)$ denotes the nonlinear function satisfying (A3) and $\bar{f}(0)=0$. Then, by using the pinning controller (7), we can derive the following theorem.

Theorem 8. Suppose that assumptions (A1)-(A3) are true; then, the controlled dynamical networks (21) can achieve the desired cluster synchronization, if there exist two $6 \mathrm{Nn} \times \mathrm{Nn}$ matrices $\Pi_{i}(i=1,2)$ making $\Pi=\left[\begin{array}{ll}\Pi_{1} & \Pi_{2}\end{array}\right], n \times n$ constant matrices $P>0, P_{i}>0, Q_{i}>0, R_{i}>0, S_{i}(i=1,2,3)$, and $M_{l}, N_{l}>0(l=1,2)$ guaranteeing $\left[\begin{array}{cc}R_{i} & S_{i} \\ * & R_{i}\end{array}\right] \geq 0$, and $n_{1} \times n_{1}$ diagonal matrices $E>0, F>0$, and $U>0$ such that the LMIs in (22) hold:

$$
\left[\begin{array}{ccc}
\Phi+\left[\mathbf{I E}\left(I_{N} \otimes \Upsilon_{i}\right)\right] \Pi^{T}+\Pi\left[\mathbf{I E}\left(I_{N} \otimes \Upsilon_{i}\right)\right]^{T} & \Pi_{1} & \Pi_{2} \\
* & -I_{N} \otimes R_{1} & -I_{N} \otimes S_{1} \\
* & * & -I_{N} \otimes R_{1}
\end{array}\right]<0, \quad i=1,2,
$$

where $\mathbf{E}$ is expressed in Denotation 1, I is expressed in Theorem 7, and

$$
\begin{aligned}
& \Omega+\bar{\mu}_{m} \Gamma P_{2} \Gamma^{T}<0, \\
& \Omega+\bar{\mu}_{m} \Gamma P_{3} \Gamma^{T}<0,
\end{aligned}
$$

$$
\begin{aligned}
& \Omega=\left[\begin{array}{cccccc}
\Omega_{11} & Q_{3} & 0 & 0 & \Omega_{15} & \Omega_{16} \\
* & \Omega_{22} & \Omega_{23} & S_{3} & 0 & 0 \\
* & * & \Omega_{33} & R_{3}-S_{3} & 0 & 0 \\
* & * & * & -P_{3}-R_{3} & 0 & 0 \\
* & * & * & * & -U & \Omega_{56} \\
* & * & * & * & * & \Omega_{66}
\end{array}\right], \\
& \Upsilon_{1}=\left[\begin{array}{cc}
\bar{\tau}_{m} I_{n} & 0_{n} \\
-I_{n} & 0_{n} \\
0_{n} & -I_{n}
\end{array}\right],
\end{aligned}
$$




$$
\begin{gathered}
\Upsilon_{2}=\left[\begin{array}{cc}
0_{n} & \bar{\tau}_{m} I_{n} \\
-I_{n} & 0_{n} \\
0_{n} & -I_{n}
\end{array}\right], \\
\Gamma=\left[\begin{array}{c}
0_{3 n \cdot n} \\
I_{n} \\
0_{2 n \cdot n}
\end{array}\right]
\end{gathered}
$$

\section{Numerical Examples}

Two numerical examples will be provided to illustrate the derived results with some typical cases.

Example 1. Consider one 2-dimensional delayed dynamical network (1) described by

with

$$
\begin{aligned}
& \Phi_{11}= L^{1} \otimes\left(M_{2} G\right)+\left(L^{1} \otimes M_{2} G\right)^{T}-2 \Xi \otimes\left(M_{1} G\right) \\
&-I_{N} \otimes\left(\tau_{0}^{2} Q_{1}\right), \\
& \Phi_{12}= {\left[\left(L^{2}\right)^{T}-\Theta\right] \otimes\left(M_{1} H\right), } \\
& \Phi_{13}= I_{N} \otimes\left(\tau_{0} Q_{1}\right), \\
& \Phi_{16}= {\left[\left(L^{1}\right)^{T}-\Xi\right] \otimes\left(M_{2} G\right)^{T}, } \\
& \Phi_{22}=-I_{N} \otimes N_{2}, \\
& \Phi_{26}= {\left[\left(L^{2}\right)^{T}-\Theta\right] \otimes\left(M_{2} H\right)^{T}, } \\
& \Phi_{33}=-I_{N} \otimes\left(Q_{1}+Q_{2}\right), \\
& \Phi_{44}=-I_{N} \otimes R_{2}, \\
& \Phi_{45}=-I_{N} \otimes S_{2}, \\
& \Phi_{55}=-I_{N} \otimes R_{2}, \\
& \Phi_{66}=-I_{N} \otimes N_{1}, \\
& \Omega_{11}= P_{1}+\tau_{0}^{2} Q_{2}+\bar{\tau}_{m}^{2} R_{2}-Q_{3}-W U \Sigma_{1} W^{T}-M_{1} C \\
&-C M_{1}^{T}, \\
& \Omega_{15}= M_{1} A+W U \Sigma_{2}, \\
& \Omega_{16}=-M_{1}+P-C M_{2}^{T}+F \Sigma^{+} W^{T}-E \Sigma^{-} W^{T}, \\
& \Omega_{22}=-P_{1}-Q_{3}-R_{3}+P_{2}, \\
& \Omega_{23}= R_{3}-S_{3}, \\
& \Omega_{33}=\left(\mu_{0}-1\right) P_{2}+\left(1-\mu_{m}\right) P_{3}-2 R_{3}+S_{3}+S_{3}^{T} \\
&-V \Sigma_{1}+N_{2}, \\
& \Omega_{56}= E^{T}-F^{T}+A^{T} M_{2}^{T}, \\
& \Omega_{1}+\tau_{0}^{2} Q_{3}+\bar{\tau}_{m}^{2} R_{3}+\frac{\tau_{0}^{4}}{4} Q_{1}+\frac{\delta_{m}^{2}}{4} R_{1}-M_{2} \\
&=N_{1},
\end{aligned}
$$

$$
\dot{x}_{i}(t)=-C x_{i}(t)+A \bar{f}\left(x_{i}(t)\right)+B \bar{f}\left(x_{i}(t-\tau(t))\right)
$$

$$
\begin{aligned}
& +u_{i}(t)+\sum_{j=1}^{4} l_{i j}^{1} G x_{j}(t)+\sum_{j=1}^{4} l_{i j}^{1} H x_{j}(t-\tau(t)) \\
& +\sum_{j=1}^{4} l_{i j}^{1} K\left(\int_{t-\tau(t)}^{t} x_{j}(s) d s\right), \quad i=1,2,3,4,
\end{aligned}
$$

with the following parameters:

$$
\begin{aligned}
C & =\left[\begin{array}{cc}
1.25 & 0 \\
0 & 1.25
\end{array}\right], \\
A & =\left[\begin{array}{cc}
1.8 & -0.15 \\
-5.2 & -3.5
\end{array}\right], \\
B & =\left[\begin{array}{ll}
-1.7 & -0.12 \\
-0.26 & -2.5
\end{array}\right], \\
G & =\left[\begin{array}{ll}
2.5 & 0.2 \\
0.2 & 0.5
\end{array}\right], \\
H & =\left[\begin{array}{cc}
0.5 & 0.25 \\
0.25 & 0.5
\end{array}\right], \\
K & =\left[\begin{array}{cc}
0.5 & 0.25 \\
0.25 & 0.5
\end{array}\right], \\
\bar{f}\left(x_{i}\right) & =\left[\begin{array}{ll}
\tanh \left(x_{i 1}\right) \\
\tanh \left(x_{i 2}\right)
\end{array}\right] .
\end{aligned}
$$

Then, through adopting the pinning controller in (7),

$$
u_{i}(t)= \begin{cases}-1.5 G\left[x_{i}(t)-s_{l}(t)\right]-0.25 H\left[x_{i}(t-\tau(t))-s_{l}(t-\tau(t))\right], & i=m_{l}, \quad l=1,2 \\ 0, & i \neq m_{l},\end{cases}
$$

with $\Xi=\operatorname{diag}(0,1.5,0,1.5)$ and $\Theta=\operatorname{diag}(0,0.25,0,0.25)$, we assume that the desired cluster synchronization states of DNNs (25) are $s_{1}(t)$ and $s_{2}(t)$, which can satisfy

$$
\dot{s}_{i}(t)=-C s_{i}(t)+A \bar{f}\left(s_{i}(t)\right)+B \bar{f}\left(s_{i}(t-\tau(t))\right),
$$$$
i=1,2 \text {, }
$$ 
TABLE 1: The calculated MAUBs $\tau_{\max }$ by setting $\tau_{0}=0, \mu_{0}=$ $-0.2,-0.1$, and unavailable $\mu_{0}$.

\begin{tabular}{lccccc}
\hline Methods & $\mu_{m}$ & 0.4 & 0.6 & 0.8 & 1.2 \\
\hline Li and Cao [30] & & 1.112 & 1.035 & 1.004 & - \\
Wang et al. [31] & -0.2 & 1.154 & 1.074 & 1.048 & 0.987 \\
Theorem 7 & & 1.243 & 1.126 & 1.100 & 1.022 \\
\hline Li and Cao [30] & & 1.112 & 1.035 & 1.004 & - \\
Wang et al. [31] & -0.1 & 1.165 & 1.084 & 1.077 & 0.996 \\
Theorem 7 & & 1.263 & 1.132 & 1.127 & 1.065 \\
\hline Li and Cao [30] & & 1.112 & 1.035 & 1.004 & - \\
Wang et al. [31] & - & 1.127 & 1.046 & 1.035 & 0.945 \\
Theorem 8 & & 1.195 & 1.094 & 1.067 & 0.996 \\
\hline
\end{tabular}

with different initial conditions. In order to reduce the number of controllers and realize the cluster synchronization, we can use the controlled networks sets $\left\{m_{1}, m_{2}\right\}=\{2,4\}$ and, respectively, choose the configuration matrices as $L^{1}=$ $\left[\begin{array}{ll}L_{11}^{1} & L_{12}^{1} \\ L_{21}^{1} & L_{22}^{1}\end{array}\right]$ with

$$
\begin{aligned}
L_{11}^{1} & =\left[\begin{array}{cc}
-1.5 & 1.5 \\
1.5 & -1.5
\end{array}\right], \\
L_{12}^{1} & =\left[\begin{array}{ll}
-1 & 1 \\
-1 & 1
\end{array}\right], \\
L_{21}^{1} & =\left[\begin{array}{ll}
0.5 & -0.5 \\
0.5 & -0.5
\end{array}\right], \\
L_{22}^{1} & =\left[\begin{array}{cc}
-2 & 2 \\
2 & -2
\end{array}\right] .
\end{aligned}
$$

In what follows, two cases will be given to illustrate the efficiency and reduced conservatism of our results.

Case 1. Given $\tau_{0}=0$, choose three inner coupling matrices of diagonal form as

$$
\begin{aligned}
& G=\left[\begin{array}{cc}
0.5 & 0 \\
0 & 0.5
\end{array}\right], \\
& H=\left[\begin{array}{cc}
0.25 & 0 \\
0 & 0.25
\end{array}\right], \\
& K=\left[\begin{array}{cc}
0.25 & 0 \\
0 & 0.25
\end{array}\right] .
\end{aligned}
$$

Then, through, respectively, setting $\mu_{0}=-0.2,-0.1$, and unavailable $\mu_{0}$, we can compute the corresponding maximum allowable upper bounds (MAUBs) in Table 1 based on Theorem 7 and Remark A.2 by resorting to Matlab LMI Toolbox.

In Table 1, the term "-" means that the corresponding value is unavailable. Based on the MAUBs in Table 1, one can verify that our results can be less conservative than some existent ones. In particular, as the inner coupling matrices
$G, H$, and $K$ are not diagonal, our theorems still can be applicable while [30,31] fail.

Case 2. Choosing $\tau(t)=0.2+0.8 \sin ^{2}(2 t)$ and the inner coupling matrices $G, H$, and $K$ as Case 1 , one can derive $\tau_{0}=0.2, \tau_{m}=1.0, \mu_{0}=-1.6$, and $\mu_{m}=1.6$. Owing to the fact that $\mu_{m}>1$ and $G, H$, and $K$ are not diagonal, the methods in $[30,31]$ fail to verify the synchronization. Yet, by resorting to Matlab LMI Toolbox, Theorem 7 can guarantee the pinning cluster synchronization and the feasible solution to LMIs in (17)-(18) can be obtained as follows:

$$
\begin{aligned}
& P_{1}=\left[\begin{array}{ll}
0.562 & 0.121 \\
0.121 & 0.532
\end{array}\right], \\
& P_{2}=\left[\begin{array}{ll}
0.343 & 0.022 \\
0.022 & 0.346
\end{array}\right] \text {, } \\
& P_{3}=\left[\begin{array}{ll}
1.206 & 0.202 \\
0.202 & 0.987
\end{array}\right] \text {, } \\
& P_{4}=\left[\begin{array}{ll}
1.633 & 0.193 \\
0.193 & 0.857
\end{array}\right] \text {, } \\
& Q_{1}=\left[\begin{array}{ll}
0.002 & 0.001 \\
0.001 & 0.001
\end{array}\right], \\
& Q_{2}=\left[\begin{array}{ll}
0.005 & 0.001 \\
0.001 & 0.012
\end{array}\right] \text {, } \\
& Q_{3}=\left[\begin{array}{ll}
1.214 & 0.314 \\
0.314 & 0.685
\end{array}\right] \text {, } \\
& R_{1}=\left[\begin{array}{ll}
1.073 & 0.511 \\
0.511 & 1.034
\end{array}\right] \text {, } \\
& R_{2}=\left[\begin{array}{ll}
1.428 & 0.544 \\
0.544 & 1.428
\end{array}\right] \text {, } \\
& R_{3}=\left[\begin{array}{ll}
1.224 & 0.124 \\
0.124 & 0.985
\end{array}\right] \text {, } \\
& S_{1}=\left[\begin{array}{ll}
0.335 & 0.050 \\
0.132 & 0.325
\end{array}\right] \text {, } \\
& S_{2}=\left[\begin{array}{ll}
0.544 & 0.224 \\
0.327 & 0.675
\end{array}\right] \text {, } \\
& S_{3}=\left[\begin{array}{ll}
0.220 & 0.091 \\
0.068 & 0.202
\end{array}\right] \text {, } \\
& M_{1}=\left[\begin{array}{cc}
1.304 & -0.101 \\
-0.114 & 0.132
\end{array}\right] \text {, } \\
& M_{2}=\left[\begin{array}{ll}
1.142 & 1.102 \\
0.786 & 0.132
\end{array}\right] \text {, }
\end{aligned}
$$




$$
\begin{aligned}
N_{1} & =\left[\begin{array}{ll}
0.562 & 0.121 \\
0.121 & 0.867
\end{array}\right], \\
N_{2} & =\left[\begin{array}{ll}
0.445 & 0.342 \\
0.342 & 0.766
\end{array}\right], \\
P & =\left[\begin{array}{ll}
0.154 & 0.105 \\
0.105 & 0.445
\end{array}\right], \\
E & =0.202 I_{2}, \\
F & =0.175 I_{2}, \\
U & =0.006 I_{2}, \\
V & =0.010 I_{2} .
\end{aligned}
$$

Example 2. In this example, we consider the well-known Chua's circuit to illustrate our synchronization results, which can be expressed as

$$
\begin{aligned}
& \dot{x}_{1}=\alpha\left[x_{2}-m_{1} x_{1}+h\left(x_{1}\right)\right], \\
& \dot{x}_{2}=x_{1}-x_{2}+x_{3}, \\
& \dot{x}_{3}=-\beta x_{2}
\end{aligned}
$$

with $h\left(x_{1}\right)=(1 / 2)\left(m_{1}-m_{0}\right)\left(\left|x_{1}+c\right|-\left|x_{1}-c\right|\right)$ and the parameters $m_{0}=-1 / 7, m_{1}=2 / 7, \alpha=9, \beta=14.286$, and $c=1$. Then, the circuit model can be represented as the Lur'e system:

$$
\begin{aligned}
\dot{x}(t)= & {\left[\begin{array}{ccc}
-\frac{18}{7} & 9 & 0 \\
1 & -1 & 1 \\
0 & -14.286 & 0
\end{array}\right] x(t) } \\
& +\left[\begin{array}{ccc}
\frac{27}{7} & 0 & 0 \\
0 & 0 & 0 \\
0 & 0 & 0
\end{array}\right] \bar{f}\left(W^{T} x(t)\right)
\end{aligned}
$$

with $W=\left[\begin{array}{lll}1 & 0 & 0\end{array}\right]^{T}$ and $\bar{f}_{1}(\xi)=(1 / 2)(|\xi+1|-|\xi-1|)$ belonging to the sector $[0,1]$. Now we consider the cluster synchronization of the dynamical networks with each node being a 2-dimensional system (33) with linear coupling as

$$
\begin{aligned}
\dot{x}_{i}(t)= & -C x_{i}(t)+A \bar{f}\left(W^{T} x_{i}(t)\right)+\sum_{j=1}^{4} l_{i j}^{1} G x_{j}(t) \\
& +\sum_{j=1}^{4} l_{i j}^{2} H x_{j}(t-\tau(t))+u_{i}(t) .
\end{aligned}
$$

In order to reduce the number of controllers and realize the cluster synchronization, we adopt the pinning controller (7) as

$$
u_{i}(t)= \begin{cases}-1.5 G\left[x_{i}(t)-s_{l}(t)\right], & i=m_{l}, \quad l=1,2, \\ 0, & i \neq m_{l}\end{cases}
$$

and the desired cluster synchronization states of (A.15) satisfy

$$
\dot{s}_{i}(t)=-C s_{i}(t)+A \bar{f}\left(W^{T} s_{i}(t)\right), \quad i=1,2
$$

with the initial conditions $s_{1}(0)=\left[\begin{array}{c}0.4 \\ -0.5\end{array}\right]$ and $s_{2}(0)=\left[\begin{array}{c}-0.2 \\ 0.3\end{array}\right]$. Now through setting $\Xi=\operatorname{diag}(0,1.5,0,1.5), G=\left[\begin{array}{ll}1.5 & 0.2 \\ 0.2 & 1.5\end{array}\right]$, and the configuration matrices as $L^{1}=\left[\begin{array}{ll}L_{11}^{1} & L_{12}^{1} \\ L_{21}^{1} & L_{22}^{1}\end{array}\right]$ with

$$
\begin{aligned}
& L_{11}^{1}=\left[\begin{array}{cc}
-1.5 & 1.5 \\
1.5 & -1.5
\end{array}\right], \\
& L_{12}^{1}=\left[\begin{array}{ll}
-1 & 1 \\
-1 & 1
\end{array}\right], \\
& L_{21}^{1}=\left[\begin{array}{ll}
0 & 0 \\
0 & 0
\end{array}\right], \\
& L_{22}^{1}=\left[\begin{array}{cc}
-2 & 2 \\
2 & -2
\end{array}\right],
\end{aligned}
$$

together with Theorem 8 and LMI in Matlab Toolbox, we can easily verify that network (34) can achieve the desired cluster synchronization, which can be further supported by the synchronization error states in Figure 1.

\section{Conclusions}

This paper has investigated the problem on pinning cluster synchronization for delayed dynamical networks with linearly hybrid coupling. Two novel conditions have been derived by employing the Lyapunov-Krasovskii stability theory. It is worth pointing out that some most recently developed techniques such as combined convex technique and triple integral LKF terms have been employed, which can help extend the application areas. The synchronization criteria are presented in the forms of LMIs, which can be checked easily by referring to Matlab LMI Toolbox. Finally, two numerical examples can illustrate the less conservatism of our theorems based on some comparing results.

\section{Appendix}

Proof of Theorem 7. Based on assumptions (A1)-(A3), we can choose the Lyapunov-Krasovskii functional as

$$
V(e(t))=V_{1}(e(t))+V_{2}(e(t))+V_{3}(e(t)),
$$



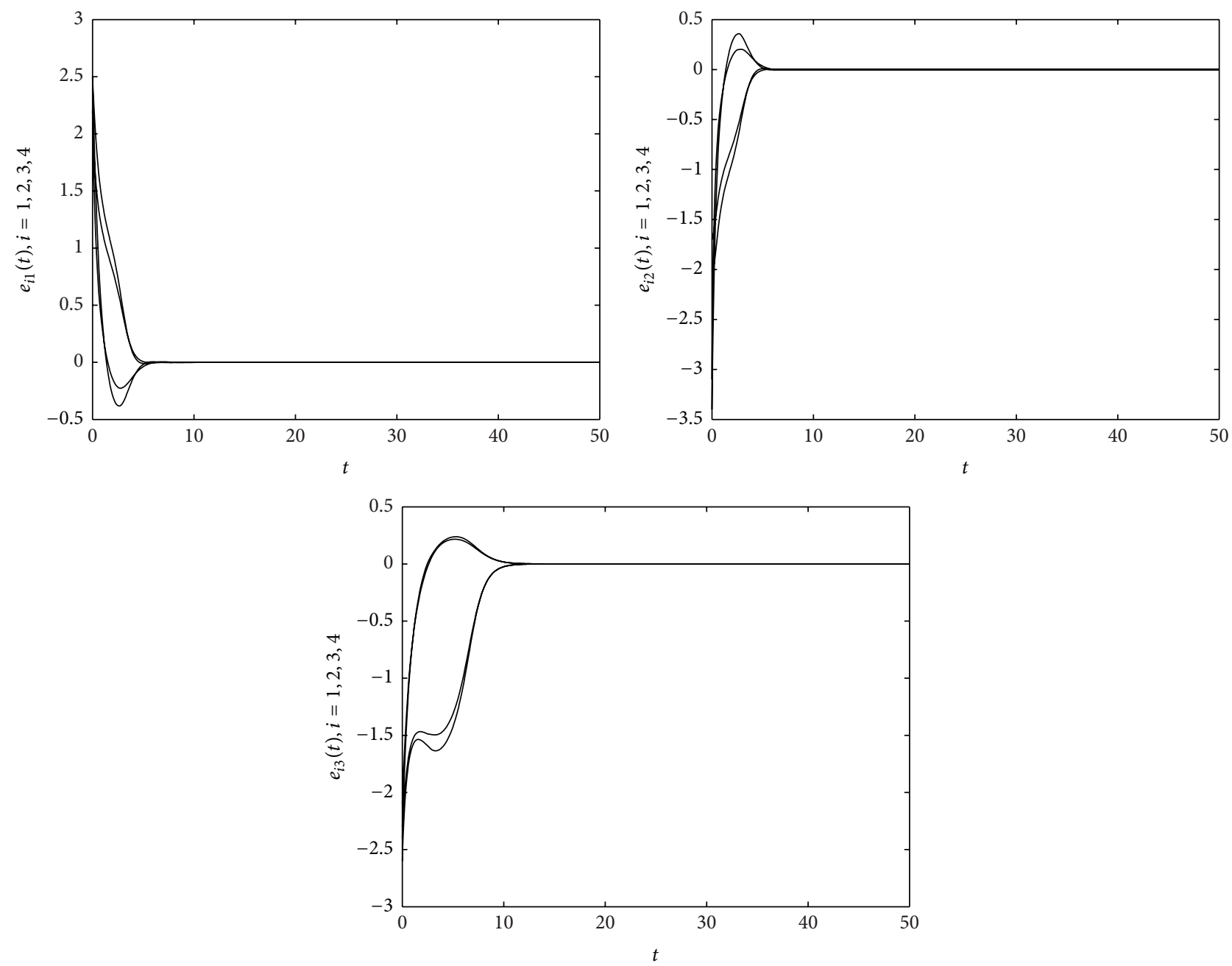

FIGURE 1: Phase and state trajectories of the error states.

where

$$
\begin{aligned}
& V_{1}(e(t))=\sum_{i=1}^{N}\left[e_{i}^{T}(t) P e_{i}(t)\right. \\
& +\int_{t-\tau(t)}^{t} f^{T}\left(e_{i}(s)\right) P_{1} f\left(e_{i}(s)\right) d s \\
& +\int_{t-\tau_{0}}^{t} e_{i}^{T}(s) P_{2} e_{i}(s) d s+\int_{t-\tau(t)}^{t-\tau_{0}} e_{i}^{T}(s) P_{3} e_{i}(s) d s \\
& +\int_{t-\tau_{m}}^{t-\tau(t)} e_{i}^{T}(s) P_{4} e_{i}(s) d s \\
& +2 \sum_{j=1}^{n} e_{j} \int_{0}^{e_{i j}(t)}\left[f_{j}(s)-\sigma_{j}^{-} s\right] d s \\
& \left.+2 \sum_{j=1}^{n} f_{j} \int_{0}^{e_{i j}(t)}\left[\sigma_{j}^{+} s-f_{j}(s)\right] d s\right],
\end{aligned}
$$

$$
\begin{aligned}
& V_{2}(e(t))=\sum_{i=1}^{N}\left[\frac{\tau_{0}^{2}}{2} \int_{-\tau_{0}}^{0} \int_{\varrho}^{0} \int_{t+\theta}^{t} \dot{e}_{i}^{T}(s) Q_{1} \dot{e}_{i}(s) d s d \theta d \varrho\right. \\
& \left.+\frac{\delta_{m}}{2} \int_{-\tau_{m}}^{-\tau_{0}} \int_{\varrho}^{0} \int_{t+\theta}^{t} \dot{e}_{i}^{T}(s) R_{1} \dot{e}_{i}(s) d s d \theta d \varrho\right], \\
& V_{3}(e(t)) \\
& =\sum_{i=1}^{N} \tau_{0} \int_{-\tau_{0}}^{0} \int_{t+\theta}^{t}\left[e_{i}^{T}(s) Q_{2} e_{i}(s)+\dot{e}_{i}^{T}(s) Q_{3} \dot{e}_{i}(s)\right] d s d \theta \\
& +\sum_{i=1}^{N} \bar{\tau}_{m} \int_{-\tau_{m}}^{-\tau_{0}} \int_{t+\theta}^{t}\left[e_{i}^{T}(s) R_{2} e_{i}(s)+\dot{e}_{i}^{T}(s) R_{3} \dot{e}_{i}(s)\right] d s d \theta
\end{aligned}
$$

with $n \times n$ constant matrices $P>0, P_{i}>0(i=1,2,3,4)$, $Q_{j}>0$, and $R_{j}>0(j=1,2,3)$ and $n \times n$ diagonal matrices $E=\operatorname{diag}\left(e_{1}, \ldots, e_{n}\right)>0$ and $F=\operatorname{diag}\left(f_{1}, \ldots, f_{n}\right)>0$ waiting to be determined. 
Then, the time derivative of $V_{i}(e(t))(i=1,2)$ along system (8) can be directly computed as

$$
\begin{aligned}
\dot{V}_{1} & (e(t))=\sum_{i=1}^{N}\left[2 e_{i}^{T}(t) P \dot{e}_{i}(t)\right. \\
+ & f^{T}\left(e_{i}(t)\right) P_{1} f\left(e_{i}(t)\right) \\
- & {[1-\dot{\tau}(t)] f^{T}\left(e_{i}(t-\tau(t))\right) P_{1} f\left(e_{i}(t-\tau(t))\right) } \\
+ & e_{i}^{T}(t) P_{2} e_{i}(t)-e_{i}^{T}\left(t-\tau_{0}\right)\left(P_{2}-P_{3}\right) e_{i}\left(t-\tau_{0}\right) \\
& -[1-\dot{\tau}(t)] e_{i}^{T}(t-\tau(t))\left(P_{3}-P_{4}\right) e_{i}(t-\tau(t)) \\
& -e_{i}^{T}\left(t-\tau_{m}\right) P_{4} e_{i}\left(t-\tau_{m}\right) \\
+ & 2 f^{T}\left(e_{i}(t)\right)(E-F) \dot{e}_{i}(t) \\
+ & \left.2 e_{i}^{T}(t)\left(F \Sigma^{+}-E \Sigma^{-}\right) \dot{e}_{i}(t)\right] \\
\dot{V}_{2} & (e(t))=\sum_{i=1}^{N}\left[\dot{e}_{i}^{T}(t)\left(\frac{\tau_{0}^{4}}{4} Q_{1}+\frac{\delta_{m}^{2}}{4} R_{1}\right) \dot{e}_{i}(t)\right. \\
& -\frac{\tau_{0}^{2}}{2} \int_{-\tau_{0}}^{0} \int_{t+\theta}^{t} \dot{e}_{i}^{T}(s) Q_{1} \dot{e}_{i}(s) d s d \theta \\
& \left.-\frac{\delta_{m}}{2} \int_{-\tau_{m}}^{-\tau_{0}} \int_{t+\theta}^{t} \dot{e}_{i}^{T}(s) R_{1} \dot{e}_{i}(s) d s d \theta\right] .
\end{aligned}
$$

Through employing Lemmas 3 and 4 and $\left[\begin{array}{ll}R_{1} & S_{1} \\ * & R_{1}\end{array}\right]>0$, we can estimate two terms in (A.4) as

$$
\begin{aligned}
& -\frac{\tau_{0}^{2}}{2} \int_{-\tau_{0}}^{0} \int_{t+\theta}^{t} \dot{e}_{i}^{T}(s) Q_{1} \dot{e}_{i}(s) d s d \theta \\
& \leq\left[\int_{-\tau_{0}}^{t} \int_{t+\theta}^{t} \dot{e}_{i}(s) d s d \theta\right]^{T} R_{1}\left[\int_{-\tau_{0}}^{t} \int_{t+\theta}^{t} \dot{e}_{i}(s) d s d \theta\right] \\
& =-\left[\tau_{0} e_{i}(t)-\int_{t-\tau_{0}}^{t} e_{i}(s) d s\right]^{T} Q_{1}\left[\tau_{0} e_{i}(t)\right. \\
& \left.-\int_{t-\tau_{0}}^{t} e_{i}(s) d s\right] \\
& -\frac{\tau_{m}^{2}-\tau_{0}^{2}}{2} \int_{-\tau_{m}}^{-\tau_{0}} \int_{t+\theta}^{t} \dot{e}_{i}^{T}(s) R_{1} \dot{e}_{i}(s) d s d \theta \\
& \quad=-\frac{\tau_{m}^{2}-\tau_{0}^{2}}{2}\left[\frac{\tau^{2}(t)-\tau_{0}^{2}}{\tau^{2}(t)-\tau_{0}^{2}} \int_{-\tau(t)}^{-\tau_{0}} \int_{t+\theta}^{t} \dot{e}_{i}^{T}(s) R_{1} \dot{e}_{i}(s) d s d \theta\right. \\
& \left.\quad+\frac{\tau_{m}^{2}-\tau^{2}(t)}{\tau_{m}^{2}-\tau^{2}(t)} \int_{-\tau_{m}}^{-\tau(t)} \int_{t+\theta}^{t} \dot{e}_{i}^{T}(s) R_{1} \dot{e}_{i}(s) d s d \theta\right] \\
& \leq-\left[\left[\tau(t)-\tau_{0}\right] e_{i}(t)-\int_{t-\tau(t)}^{t-\tau_{0}} e_{i}(s) d s\right]^{T} \\
& \quad \cdot R_{1}\left[\left[\tau(t)-\tau_{0}\right] e_{i}(t)-\int_{t-\tau(t)}^{t-\tau_{0}} e_{i}(s) d s\right]
\end{aligned}
$$

$$
\begin{aligned}
& -\left[\left[\tau(t)-\tau_{0}\right] e_{i}(t)-\int_{t-\tau(t)}^{t-\tau_{0}} e_{i}(s) d s\right]^{T}\left(2 S_{1}\right) \\
& \cdot\left[\left[\tau_{m}-\tau(t)\right] e_{i}(t)-\int_{t-\tau_{m}}^{t-\tau(t)} e_{i}(s) d s\right] \\
& -\left[\left[\tau_{m}-\tau(t)\right] e_{i}(t)-\int_{t-\tau_{m}}^{t-\tau(t)} e_{i}(s) d s\right]^{T} \\
& \cdot R_{1}\left[\left[\tau_{m}-\tau(t)\right] e_{i}(t)-\int_{t-\tau_{m}}^{t-\tau(t)} e_{i}(s) d s\right] \\
& =-\left[\begin{array}{l}
e_{i}(t) \\
\phi_{i}(t) \\
\psi_{i}(t)
\end{array}\right]^{T} \\
& \cdot\left[\begin{array}{cc}
\alpha(t) I_{n} & \beta(t) I_{n} \\
-I_{n} & 0 \\
0 & -I_{n}
\end{array}\right]\left[\begin{array}{ll}
R_{1} & S_{1} \\
* & R_{1}
\end{array}\right]\left[\begin{array}{cc}
\alpha(t) I_{n} & \beta(t) I_{n} \\
-I_{n} & 0 \\
0 & -I_{n}
\end{array}\right]^{T} \\
& \cdot\left[\begin{array}{l}
e_{i}(t) \\
\phi_{i}(t) \\
\psi_{i}(t)
\end{array}\right]
\end{aligned}
$$

with denoting

$$
\begin{aligned}
\alpha(t) & =\tau(t)-\tau_{0}, \\
\beta(t) & =\tau_{m}-\tau(t), \\
\phi_{i}(t) & =\int_{t-\tau(t)}^{t-\tau_{0}} e_{i}(s) d s, \\
\psi_{i}(t) & =\int_{t-\tau_{m}}^{t-\tau(t)} e_{i}(s) d s .
\end{aligned}
$$

Furthermore, together with Lemmas 3 and 4 and $\left[\begin{array}{cc}R_{i} & S_{i} \\ * & R_{i}\end{array}\right]>$ $0(i=2,3)$, we can compute

$$
\begin{gathered}
\dot{V}_{3}(e(t)) \leq \sum_{i=1}^{N}\left\{e_{i}^{T}(t)\left(\tau_{0}^{2} Q_{2}+\bar{\tau}_{m}^{2} R_{2}\right) e_{i}(t)+\dot{e}_{i}^{T}(t)\right. \\
\cdot\left(\tau_{0}^{2} Q_{3}+\bar{\tau}_{m}^{2} R_{3}\right) \dot{e}_{i}(t)-\left(\int_{t-\tau_{0}}^{t} e_{i}(s) d s\right)^{T} \\
\cdot Q_{2}\left(\int_{t-\tau_{0}}^{t} e_{i}(s) d s\right)-\left(\int_{t-\tau(t)}^{t-\tau_{0}} e_{i}(s) d s\right)^{T} \\
\cdot R_{2}\left(\int_{t-\tau(t)}^{t-\tau_{0}} e_{i}(s) d s\right)-2\left(\int_{t-\tau(t)}^{t-\tau_{0}} e_{i}(s) d s\right)^{T} \\
\cdot S_{2}\left(\int_{t-\tau_{m}}^{t-\tau(t)} e_{i}(s) d s\right)-\left(\int_{t-\tau_{m}}^{t-\tau(t)} e_{i}(s) d s\right)^{T} \\
\cdot R_{2}\left(\int_{t-\tau_{m}}^{t-\tau(t)} e_{i}(s) d s\right)-\left[e_{i}(t)-e_{i}\left(t-\tau_{0}\right)\right]^{T}
\end{gathered}
$$




$$
\begin{aligned}
& \cdot Q_{3}\left[e_{i}(t)-e_{i}\left(t-\tau_{0}\right)\right] \\
& -\left[e_{i}\left(t-\tau_{0}\right)-e_{i}(t-\tau(t))\right]^{T} \\
& \cdot R_{3}\left[e_{i}\left(t-\tau_{0}\right)-e_{i}(t-\tau(t))\right] \\
& -2\left[e_{i}\left(t-\tau_{0}\right)-e_{i}(t-\tau(t))\right]^{T} \\
& \cdot S_{3}\left[e_{i}(t-\tau(t))-e_{i}\left(t-\tau_{m}\right)\right] \\
& -\left[e_{i}(t-\tau(t))-e_{i}\left(t-\tau_{m}\right)\right]^{T} \\
& \left.\cdot R_{3}\left[e_{i}(t-\tau(t))-e_{i}\left(t-\tau_{m}\right)\right]\right\} .
\end{aligned}
$$

For any $n \times n$ constant matrices $M_{i}(i=1,2)$ and $N_{j}>0(j=$ $1,2)$, it follows from (8) and Denotation 2 that

$$
\begin{aligned}
0= & 2 \sum_{i=1}^{N}\left[e_{i}^{T}(t) M_{1}+\dot{e}_{i}^{T}(t) M_{2}\right]\left[-\dot{e}_{i}(t)-C e_{i}(t)\right. \\
& \left.+A f\left(e_{i}(t)\right)+B f\left(e_{i}(t-\tau(t))\right)\right]-2 e^{T}(t) \\
& \cdot\left[\left(\Xi \otimes M_{1} G\right) e(t)+\left(\Theta \otimes M_{1} H\right) e(t-\tau(t))\right] \\
& -2 \dot{e}^{T}(t)\left[\left(\Xi \otimes M_{2} G\right) e(t)\right. \\
& \left.+\left(\Theta \otimes M_{2} H\right) e(t-\tau(t))\right]+2 e^{T}(t) \\
& \cdot\left[\left(L^{1} \otimes M_{1} G\right) e(t)+\left(L^{2} \otimes M_{1} H\right) e(t-\tau(t))\right. \\
& \left.+\left(L^{3} \otimes M_{1} K\right)\left(\int_{t-\tau(t)}^{t} e(s) d s\right)\right]+2 \dot{e}^{T}(t) \\
& \cdot\left[\left(L^{1} \otimes M_{2} G\right) e(t)+\left(L^{2} \otimes M_{2} H\right) e(t-\tau(t))\right. \\
& \left.+\left(L^{3} \otimes M_{2} K\right)\left(\int_{t-\tau(t)}^{t} e(s) d s\right)\right]
\end{aligned}
$$

$$
\begin{aligned}
0 & =\sum_{i=1}^{N}\left[\begin{array}{c}
\dot{e}_{i}(t) \\
e_{i}(t-\tau(t))
\end{array}\right]^{T}\left[\begin{array}{cc}
N_{1} & 0 \\
0 & N_{2}
\end{array}\right]\left[\begin{array}{c}
\dot{e}_{i}(t) \\
e_{i}(t-\tau(t))
\end{array}\right] \\
& -\left[\begin{array}{c}
\dot{e}(t) \\
e(t-\tau(t))
\end{array}\right] \\
& \cdot\left[\begin{array}{cc}
I_{N} \otimes N_{1} & 0 \\
0 & I_{N} \otimes N_{2}
\end{array}\right]\left[\begin{array}{c}
\dot{e}(t) \\
e(t-\tau(t))
\end{array}\right] .
\end{aligned}
$$

Together with (A3) and any $n \times n$ diagonal matrices $U>0$ and $V>0$, one can easily derive

$$
\begin{aligned}
0 \leq & -\sum_{i=1}^{N}\left\{\left[e_{i}^{T}(t) U \Sigma_{1} e_{i}(t)-2 e_{i}^{T}(t) U \Sigma_{2} f\left(e_{i}(t)\right)\right.\right. \\
& \left.+f^{T}\left(e_{i}(t)\right) U f\left(e_{i}(t)\right)\right] \\
& +\left[e_{i}^{T}(t-\tau(t)) V \Sigma_{1} e_{i}(t-\tau(t))\right. \\
& -2 e_{i}^{T}(t-\tau(t)) V \Sigma_{2} f\left(e_{i}(t-\tau(t))\right) \\
& \left.\left.+f^{T}\left(e_{i}(t-\tau(t))\right) V f\left(e_{i}(t-\tau(t))\right)\right]\right\} .
\end{aligned}
$$

Then, through employing Denotations 1 and 2 and the right terms in (A.3), (A.4), (A.5), (A.7), (A.8), and (A.9), the term $\dot{V}(e(t))$ can be estimated as

$$
\begin{aligned}
& \dot{V}(e(t)) \leq \sum_{i=1}^{N} \zeta_{i}^{T}(t)\left[\Omega+\left[\dot{\tau}(t)-\mu_{0}\right] \Gamma_{1}\left[\begin{array}{cc}
P_{1} & 0 \\
0 & P_{3}
\end{array}\right] \Gamma_{1}^{T}\right. \\
& \left.+\left[\mu_{m}-\dot{\tau}(t)\right] \Gamma_{2} P_{4} \Gamma_{2}^{T}\right] \zeta_{i}(t)+\xi^{T}(t)[\Phi \\
& \left.\quad-\operatorname{IE}\left(I_{N} \otimes \Upsilon(t)\left[\begin{array}{ll}
R_{1} & S_{1} \\
* & R_{1}
\end{array}\right] \Upsilon^{T}(t)\right)(\mathbf{I E})^{T}\right] \xi(t),
\end{aligned}
$$

where $\Upsilon^{T}(t)=\left[\begin{array}{ccc}\alpha(t) I_{n} & -I_{n} & 0_{n} \\ \beta(t) I_{n} & 0_{n} & -I_{n}\end{array}\right]$, the denotations $\Phi, \Omega, \mathbf{I}, \Gamma_{1}$, and $\Gamma_{2}$ are expressed in (17)-(18), and

$$
\begin{aligned}
& \xi^{T}(t)=\left[e^{T}(t) e^{T}(t-\tau(t))\left(\int_{t-\tau_{0}}^{t} e(s) d s\right)^{T}\left(\int_{t-\tau(t)}^{t-\tau_{0}} e(s) d s\right)^{T}\left(\int_{t-\tau_{m}}^{t-\tau(t)} e(s) d s\right)^{T} \dot{e}^{T}(t)\right],
\end{aligned}
$$

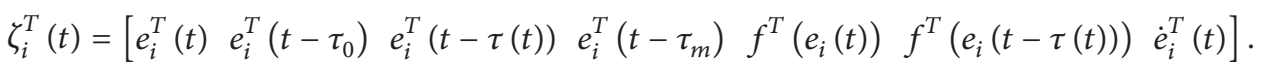

Now as for the terms in (A.10), if the two following inequalities

$$
\begin{gathered}
\Omega+\left(\dot{\tau}(t)-\mu_{0}\right) \Gamma_{1}\left[\begin{array}{ll}
P_{1} & 0 \\
0 & P_{3}
\end{array}\right] \Gamma_{1}^{T} \\
+\left(\mu_{m}-\dot{\tau}(t)\right) \Gamma_{2} P_{4} \Gamma_{2}^{T}<0,
\end{gathered}
$$

$$
\Phi-\operatorname{IE}\left(I_{N} \otimes \Upsilon(t)\left[\begin{array}{cc}
R_{1} & S_{1} \\
* & R_{1}
\end{array}\right] \Upsilon^{T}(t)\right)(\mathbf{I E})^{T}<0
$$

can be true simultaneously, then we can obtain $\dot{V}(e(t))<0$ for any $e(t) \neq 0$.

Together with Lemma 5, the LMIs in (18) can make $\Omega+\left[\dot{\tau}(t)-\mu_{0}\right] \Gamma_{1}\left[\begin{array}{cc}P_{1} & 0 \\ 0 & P_{3}\end{array}\right] \Gamma_{1}^{T}+\left[\mu_{m}-\dot{\tau}(t)\right] \Gamma_{2} P_{4} \Gamma_{2}^{T}<0$ hold. 
Meanwhile, with the denotations $\Upsilon_{1}$ and $\Upsilon_{2}$ in (17), the LMI results in (17) mean the following inequalities

$$
\begin{aligned}
& \Phi+\mathbf{I E}\left(I_{N} \otimes \Upsilon_{1}\right) \Pi^{T}+\Pi\left(I_{N} \otimes \Upsilon_{1}^{T}\right)(\mathbf{I E})^{T}<0, \\
& \Phi+\mathbf{I E}\left(I_{N} \otimes \Upsilon_{2}\right) \Pi^{T}+\Pi\left(I_{N} \otimes \Upsilon_{2}^{T}\right)(\mathbf{I E})^{T}<0
\end{aligned}
$$

to be true. Then, based on Lemma 5, the terms $\Phi+\operatorname{IE}\left(I_{N} \otimes\right.$ $\left.\Upsilon_{i}\right) \Pi^{T}+\Pi\left(I_{N} \otimes \Upsilon_{i}^{T}\right)(\mathbf{I E})^{T}<0(i=1,2)$ in (A.13) can guarantee

$$
\begin{aligned}
\Phi+ & {\left[\operatorname{IE}\left(I_{N} \otimes \Upsilon(t)\right)\right] \Pi^{T}+\Pi\left[\operatorname{IE}\left(I_{N} \otimes \Upsilon(t)\right)\right]^{T} } \\
& <0 .
\end{aligned}
$$

Furthermore, one can easily check

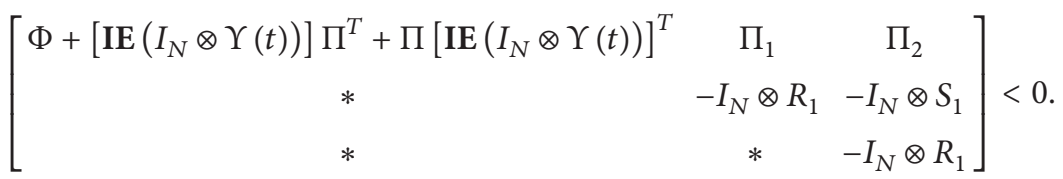

Then, based on Lemma 6, the matrix inequality in (A.15) can make the following term true:

$$
\begin{aligned}
\Phi- & {\left[\operatorname{IE}\left(I_{N} \otimes \Upsilon(t)\right)\right] } \\
& {\left[\begin{array}{cc}
I_{N} \otimes R_{1} & I_{N} \otimes S_{1} \\
* & I_{N} \otimes R_{1}
\end{array}\right]\left[\operatorname{IE}\left(I_{N} \otimes \Upsilon(t)\right)\right]^{T}<0 . }
\end{aligned}
$$

Therefore, the LMIs in (17)-(18) can make $\dot{V}(e(t))<0$ for any $e(t) \neq 0$. Then, based on Lyapunov stability theory and Definition 2, the error system (8) is asymptotically stable; that is, the controlled networks (1) can achieve the desired cluster synchronization.

Remark A.1. During the proof procedure in Theorem 7, one can easily check that, firstly, two triple integral Lyapunov terms in $V_{2}(e(t))$ could play an essential role in reducing the conservatism, and some tighter upper bounds on the derivatives were presented; secondly, we combined reciprocal convex technique with normal convex one to tackle time-delay issue; thirdly, through employing the Kronecker product in (A.10), (A.13), and (A.15), the forms of the inner coupling matrices $G, H$, and $K$ can be described more generally than the ones in $[30,31]$.

Remark A.2. As for time-delay issue, most present works on delayed dynamical networks have only considered the case $\dot{\tau}(t) \leq \mu_{m}$. Thus, through replacing $\int_{t-\tau_{m}}^{t-\tau(t)} e_{i}^{T}(s) P_{4} e_{i}(s) d s$ by $\int_{t-\tau_{m}}^{t-\tau_{0}} e_{i}^{T}(s) P_{4} e_{i}(s) d s$ in (A.1) and using the similar proving procedure, we also can derive some relevant results.

Proof of Theorem 8. Based on assumptions (A1)-(A3), we can choose the Lyapunov-Krasovskii functional as

$$
V(e(t))=V_{1}(e(t))+V_{2}(e(t))+V_{3}(e(t)),
$$

where

$$
\begin{aligned}
& V_{1}(e(t))=\sum_{i=1}^{N}\left[e_{i}^{T}(t) P e_{i}(t)+\int_{t-\tau_{0}}^{t} e_{i}^{T}(s) P_{1} e_{i}(s) d s\right. \\
& +\int_{t-\tau(t)}^{t-\tau_{0}} e_{i}^{T}(s) P_{2} e_{i}(s) d s \\
& +\int_{t-\tau_{m}}^{t-\tau(t)} e_{i}^{T}(s) P_{3} e_{i}(s) d s \\
& +2 \sum_{j=1}^{n_{1}} e_{j} \int_{0}^{W_{i}^{T} e_{j}(t)}\left[f_{j}(s)-\sigma_{j}^{-} s\right] d s \\
& \left.+2 \sum_{j=1}^{n_{1}} f_{j} \int_{0}^{W_{i}^{T} e_{j}(t)}\left[\sigma_{j}^{+} s-f_{j}(s)\right] d s\right]
\end{aligned}
$$

with setting $n \times n$ diagonal matrices $E=\operatorname{diag}\left(e_{1}, \ldots, e_{n_{1}}\right)>0$, $F=\operatorname{diag}\left(f_{1}, \ldots, f_{n_{1}}\right)>0$, and $V_{i}(e(t))(i=2,3)$ identical to the ones in Theorem 7 .

Remark A.3. Theorems 7 and 8 provide two less conservative criteria that ensure the cluster synchronization of network models (1) and (28) via pinning control, which can be easily checked by resorting to LMI in the Matlab Toolbox and do not require the inner coupling matrices to be of diagonal form. Moreover, as for $h=1$, the derived theorems can be reduced to guarantee the pinning global synchronization.

Remark A.4. Presently, the reciprocal convex technique in [37] has been widely put forward to tackle time-delay systems, owing to the fact that it could reduce the conservatism more efficiently than some previous ones [23, 31]. Yet it comes to our attention that the reciprocal technique has not been utilized to tackle the pinning cluster synchronization on delayed complex networks and it has been fully considered in this work.

\section{Competing Interests}

The authors declare that they have no competing interests. 


\section{Acknowledgments}

This research is supported by the National Natural Science Foundation of China (nos. 61374116 and 61473079), Jiangsu Natural Science Foundation (no. BK20150888), and Natural Science Foundation for Jiangsu's Universities (no. 15KJB12004).

\section{References}

[1] L. M. Pecora and T. L. Carroll, "Synchronization in chaotic systems," Physical Review Letters, vol. 64, article 821, 1990.

[2] T. L. Carroll and L. M. Pecora, "Synchronizing chaotic circuits," IEEE Transactions on Circuits and Systems-I, vol. 38, no. 4, pp. 453-456, 1991.

[3] X.-Y. Guo and J.-M. Li, "Stochastic synchronization for timevarying complex dynamical networks," Chinese Physics $B$, vol. 21, no. 2, Article ID 020501, 2012.

[4] Q.-J. Zhang and J.-C. Zhao, "Novel criteria for exponential synchronization of inner time-varying complex networks with coupling delay," Chinese Physics B, vol. 21, no. 4, Article ID 040502, 2012.

[5] H.-X. Qin, J. Ma, W.-Y. Jin, and C.-N. Wang, "Dislocation coupling-induced transition of synchronization in two-layer neuronal networks," Communications in Theoretical Physics, vol. 62, no. 5, article 755, 2014.

[6] B. Y. Zhang, J. Lam, and S. Y. Xu, "Relaxed results on reachable set estimation of time-delay systems with bounded peak inputs," International Journal of Robust and Nonlinear Control, vol. 26, no. 9, pp. 1994-2007, 2016.

[7] B. Y. Zhang, J. Lam, and S. Y. Xu, "Stability analysis of distributed delay neural networks based on relaxed LyapunovKrasovskii Functionals," IEEE Transactions on Neural Networks and Learning Systems, vol. 26, no. 7, pp. 1480-1492, 2015.

[8] S.-G. Wang and H.-X. Yao, "Impulsive synchronization of two coupled complex networks with time-delayed dynamical nodes," Chinese Physics B, vol. 20, no. 9, Article ID 090513, 2011.

[9] X. Y. Meng and T. W. Chen, "Event based agreement protocols for multi-agent networks," Automatica, vol. 49, no. 7, pp. 21252132, 2013.

[10] T. Li, A. G. Song, S. M. Fei, and T. Wang, "Global synchronization in arrays of coupled Lurie systems with both time delay and hybrid coupling," Communications in Nonlinear Science and Numerical Simulations, vol. 16, no. 1, pp. 10-20, 2011.

[11] T. Li, T. Wang, X. Yang, and S. Fei, "Pinning cluster synchronization for delayed dynamical networks via Kronecker product," Circuits, Systems, and Signal Processing, vol. 32, no. 4, pp. 19071929, 2013.

[12] K. Ratnavelu, M. Manikandan, and P. Balasubramaniam, "Synchronization of fuzzy bidirectional associative memory neural networks with various time delays," Applied Mathematics and Computation, vol. 270, pp. 582-605, 2015.

[13] H. R. Karimi and H. Gao, "New delay-dependent exponential $H_{\infty}$ synchronization for uncertain neural networks with mixed time delays," IEEE Transactions on Systems, Man, and Cybernetics, Part B: Cybernetics, vol. 40, no. 1, pp. 173-185, 2010.

[14] B. S. Chen, C. H. Chiang, and S. K. Nguang, "Robust $H_{\infty}$ synchronization design of nonlinear coupled network via fuzzy interpolation method," IEEE Transactions on Circuits and Systems-I, vol. 58, no. 2, pp. 349-362, 2011.
[15] W. L. He and J. D. Cao, "Exponential synchronization of hybrid coupled networks with delayed coupling," IEEE Transactions on Neural Networks, vol. 21, no. 4, pp. 571-583, 2010.

[16] M. J. Park, O. M. Kwon, J. H. Park, S. M. Lee, and E. J. Cha, "Synchronization criteria for coupled Hopfield neural networks with time-varying delays," Chinese Physics B, vol. 20, no. 11, Article ID 110504, 2011.

[17] P. Balasubramaniam, M. Kalpana, and R. Rakkiyappan, "Linear matrix inequality approach for synchronization control of fuzzy cellular neural networks with mixed time delays," Chinese Physics B, vol. 21, no. 4, Article ID 048402, 2012.

[18] L. Zhou, Z. Wang, J. Zhou, and W. Zhou, "Mean square synchronization of neural networks with Lévy noise via sampleddata and actuator saturating controller," Neurocomputing, vol. 173, part 3, pp. 1235-1244, 2016.

[19] H. G. Zhang, J. Y. Wang, Z. S. Wang, and H. J. Liang, "Mode-dependent stochastic synchronization for Markovian coupled neural networks with time-varying mode-delays," IEEE Transactions on Neural Networks and Learning Systems, vol. 26, no. 11, pp. 2621-2634, 2015.

[20] D. B. Tong, W. N. Zhou, X. H. Zhou, J. Yang, L. Zhang, and Y. Xu, "Exponential synchronization for stochastic neural networks with multi-delayed and Markovian switching via adaptive feedback control," Communications in Nonlinear Science and Numerical Simulation, vol. 29, no. 1-3, pp. 359-371, 2015.

[21] J. D. Cao and L. L. Li, "Cluster synchronization in an array of hybrid coupled neural networks with delay," Neural Networks, vol. 22, no. 4, pp. 335-342, 2009.

[22] L.-P. Deng and Z.-Y. Wu, "Impulsive cluster synchronization in community network with nonidentical nodes," Communications in Theoretical Physics, vol. 58, no. 4, pp. 525-530, 2012.

[23] T. Li, T. Wang, A.-G. Song, and S.-M. Fei, "Exponential synchronization for arrays of coupled neural networks with timedelay couplings," International Journal of Control, Automation and Systems, vol. 9, no. 1, pp. 187-196, 2011.

[24] Z. Y. Wang, L. H. Huang, Y. N. Wang, and Y. Zuo, "Synchronization analysis of networks with both delayed and non-delayed couplings via adaptive pinning control method," Communications in Nonlinear Science and Numerical Simulation, vol. 15, no. 12, pp. 4202-4208, 2010.

[25] S. M. Cai, J. J. Hao, Q. B. He, and Z. R. Liu, "Exponential synchronization of complex delayed dynamical networks via pinning periodically intermittent control," Physics Letters A, vol. 375, no. 19, pp. 1965-1971, 2011.

[26] H. F. Zhang, K. Z. Li, and X. C. Fu, "On pinning control of some typical discrete-time dynamical networks," Communications in Nonlinear Science and Numerical Simulation, vol. 15, no. 2, pp. 182-188, 2010.

[27] X. W. Liu and T. P. Chen, "Synchronization of nonlinear coupled networks via aperiodically intermittent pinning control," IEEE Transactions on Neural Networks and Learning Systems, vol. 26, no. 1, pp. 113-126, 2015.

[28] C. Zheng and J. D. Cao, "Robust synchronization of coupled neural networks with mixed delays and uncertain parameters by intermittent pinning control," Neurocomputing, vol. 141, pp. 153-159, 2014.

[29] X. W. Liu and Y. Xu, "Cluster synchronization in complex networks of nonidentical dynamical systems via pinning control," Neurocomputing, vol. 168, pp. 260-268, 2015.

[30] L. L. Li and J. D. Cao, "Cluster synchronization in an array of coupled stochastic delayed neural networks via pinning control," Neurocomputing, vol. 74, no. 5, pp. 846-856, 2011. 
[31] T. Wang, T. Li, X. Yang, and S. Fei, "Cluster synchronization for delayed Lur'e dynamical networks based on pinning control," Neurocomputing, vol. 83, pp. 72-82, 2012.

[32] C. Hua, C. Ge, and X. Guan, "Synchronization of chaotic lur'e systems with time delays using sampled-data control," IEEE Transactions on Neural Networks and Learning Systems, vol. 26, no. 6, pp. 1214-1221, 2015.

[33] X. Meng, J. Lam, B. Du, and H. Gao, "A delay-partitioning approach to the stability analysis of discrete-time systems," Automatica, vol. 46, no. 3, pp. 610-614, 2010.

[34] E. Vaadia and A. Acrtsen, "Coding and computation in the cortex: single-neuron activity and cooperative phenomena," in Information Processing in the Cortex: Experiments and Theory, vol. 21, pp. 81-121, Springer, Berlin, Germany, 1992.

[35] J. Sun, G. P. Liu, J. Chen, and D. Rees, "Improved delay-rangedependent stability criteria for linear systems with time-varying delays," Automatica, vol. 46, no. 2, pp. 466-470, 2010.

[36] O. M. Kwon, S. M. Lee, J. H. Park, and E. J. Cha, "New approaches on stability criteria for neural networks with interval time-varying delays," Applied Mathematics and Computation, vol. 218, no. 19, pp. 9953-9964, 2012.

[37] J. Liu and J. Zhang, "Note on stability of discrete-time timevarying delay systems," IET Control Theory and Applications, vol. 6, no. 2, pp. 335-339, 2012. 


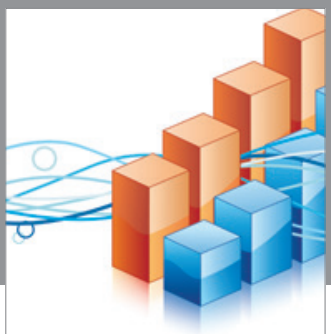

Advances in

Operations Research

vatem alat4

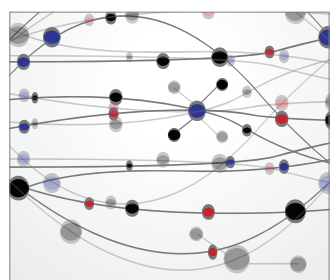

\section{The Scientific} World Journal
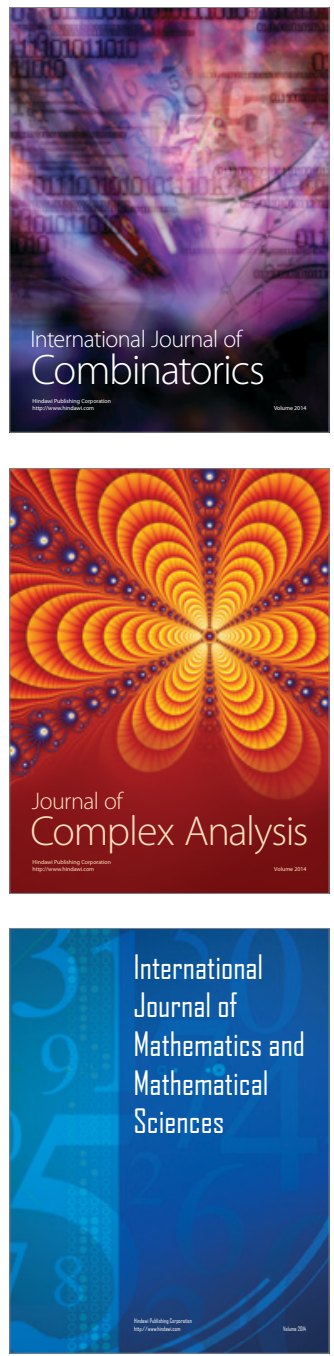
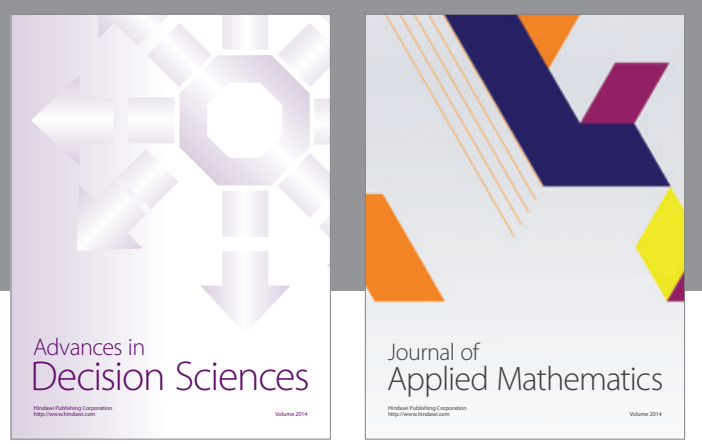

Algebra

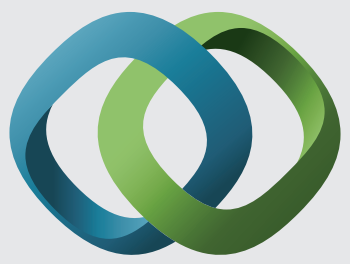

\section{Hindawi}

Submit your manuscripts at

http://www.hindawi.com
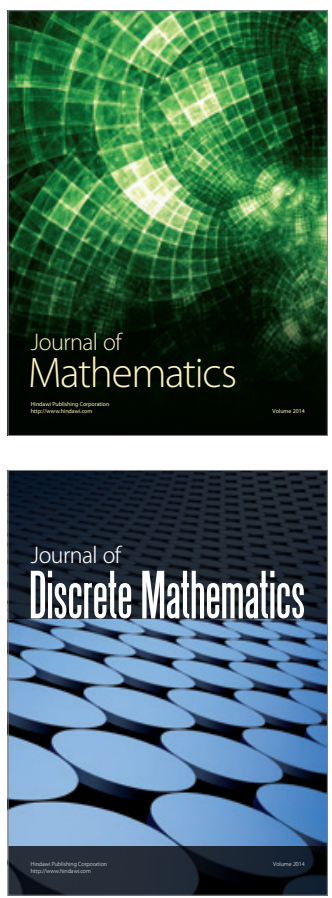

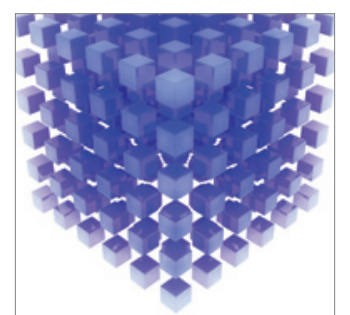

Mathematical Problems in Engineering
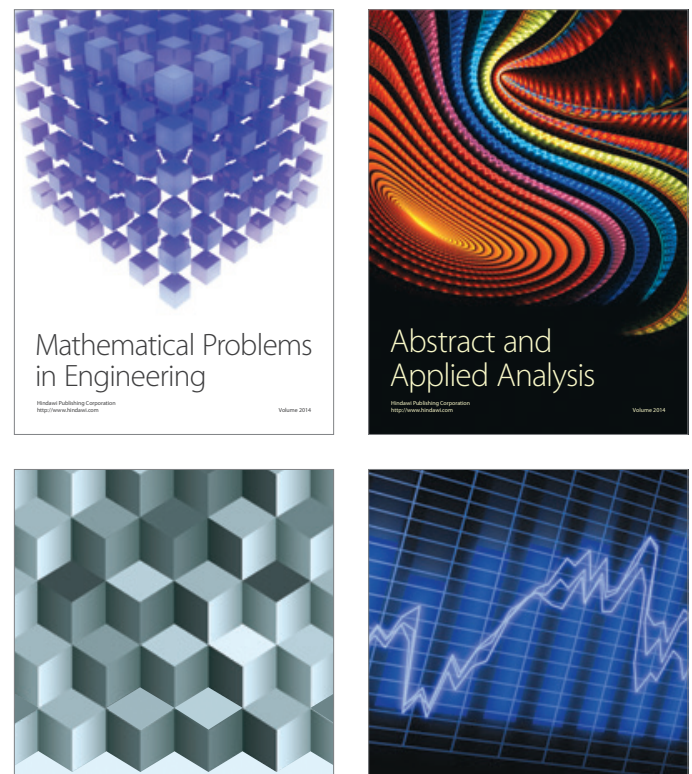

Journal of

Function Spaces

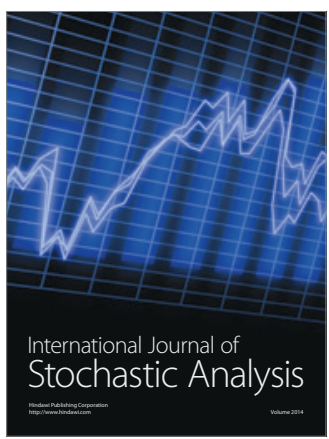

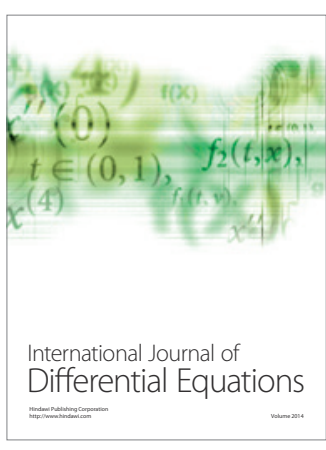
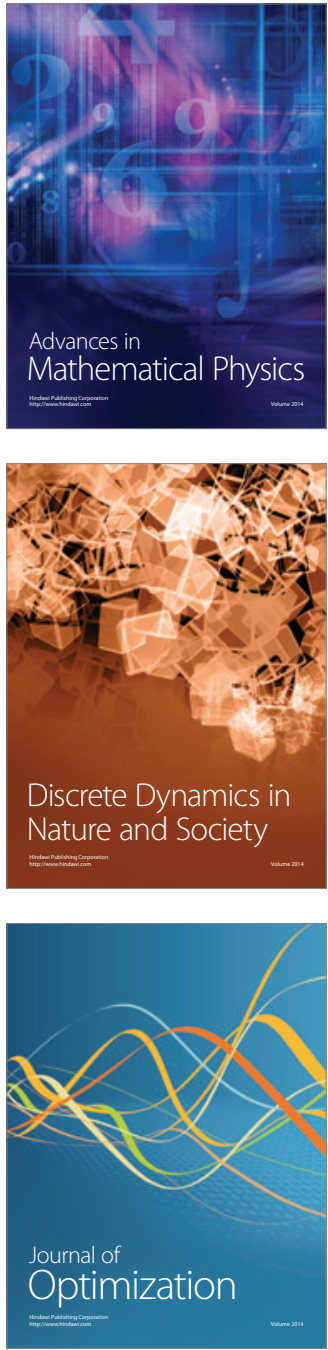